\title{
ON COMPACT TOPOLOGICAL LATTICES OF FINITE DIMENSION
}

\author{
BY \\ TAE HO CHOE
}

Introduction. In [11], Kaplansky proved that a compact semisimple topological ring is isomorphic and homeomorphic with a cartesian direct sum of finite simple rings; this implies that any compact Boolean topological lattice is always totally disconnected. However, in the proof of his theorem, Kaplansky utilized duality theorem in the sense of a topological group. Professor A. D. Wallace had suggested the possibility of a proof of the latter theorem (Boolean ring case) which is independent of the duality theorem. In $\S 1$, we show, without using the duality theorem, that any compact Boolean topological lattice of finite dimension is always totally disconnected. However, with the use of duality theorem, this theorem can be generalized as follows: a locally compact and locally convex Boolean topological lattice is totally disconnected. In the remainder of this section, we shall exhibit a sufficient condition for a compact complemented modular topological lattice to be totally disconnected.

A. D. Wallace [14] conjectured that the center of a compact connected topological lattice $L$ of dimension $n$ contains at most $2^{n}$ elements. L. W. Anderson [2] has shown that if $L$ is distributive, then Wallace's conjecture is true. In $\$ 2$, we prove that the conjecture is always true. We shall show some necessary and sufficient conditions for a topological lattice to be isomorphic and homeomorphic with the Euclidean $n$-cell. In addition, several structure theorems of compact topological lattice are given. After introducing the definition of the ordinal sum of topological lattices, we shall find a necessary and sufficient condition for a compact connected distributive topological lattice to be decomposed into a finite ordinal sum of finite cartesian products of closed connected chains.

The author wishes to express his gratitude to Professor A. D. Wallace for his helpful suggestions and kind criticisms.

Preliminaries. The lattice operations of join and meet are designated by $\vee$ and $\wedge$, respectively. Set operations are indicated by rounded symbols: $\cap, \cup$ and $\subset$ stand for intersection, union and inclusion, respectively. The empty set is denoted by $\square$. We define a topological lattice to be a pair $(L, \tau)$ where $L$ is a lattice, and is a Hausdorff space under the topology $\tau$ in which the lattice operations $\wedge$ and $\vee$ are continuous.

For a pair of subsets $A$ and $B$ of $L$, we shall use $A \wedge B$ and $A \vee B$ to denote the

Received by the editors March 23, 1967. 
sets $\{a \wedge b \mid a \in A$ and $b \in B\}$ and $\{a \vee b \mid a \in A$ and $b \in B\}$, respectively. For a subset $A$ of $L, A^{*}, A^{\circ}$ and $F(A)=A^{*} \backslash A^{\circ}$ denote the closure, the interior and the boundary of $A$, respectively. We use the term iseomorphism to mean a mapping which is simultaneously an isomorphism and homeomorphism.

$H^{n}(X)$ will denote the $n$-dimensional Alexander-Kolmogoroff cohomology group of the topological space $X$ with coefficients in some nontrivial abelian group $G$ [9].

We shall use the following Cohen's codimension definition [7]. Define $X \in D^{-1}(G)$ if $X=\square$, and $X \in D^{n}(G)$ for $n \geqq 0$ if and only if $X$ is a locally compact Hausdorff space such that if $A \subset X$ is compact, if $B=B^{*} \subset A$, and if the inclusion mapping $i: B \subset A$, then the induced mapping $i^{*}: H^{n}(A) \rightarrow H^{n}(B)$ is an epimorphism. The g.l.b. of $\left\{n \mid X \in D^{n}(G)\right\}$ is called the codimension of the space $X$, denoted by $\operatorname{cd}(X)$.

It is known [1] that if $L$ is a topological lattice and if $A$ is a compact subset of $L$, then $A \wedge L$ and $A \vee L$ are both closed.

1. Total disconnectedness in a compact topological lattice. Let $L$ be a lattice. We recall that the breadth [6] of $L$, denoted by $\operatorname{Br}(L)$, is the smallest integer $n$ such that any finite subset $F$ of $L$ has a subset $F^{\prime}$ of, at most, $n$ elements such that Inf $F=\operatorname{Inf} F^{\prime}$. If $x$ and $y$ are elements of a lattice $L$ with 0 , and if $x \wedge y=0$, then $x$ and $y$ are termed disjoint elements.

Now we shall prove the following theorem:

1.1 THEOREM. If $L$ is a nondegenerate compact connected distributive topological lattice, and if every nonunit element has a nonzero disjoint element in $L$, then the codimension of $L$ is infinite.

Before the proof of the theorem, we review some results which will be needed in the proof. E. Dyer and A. Shields [8] have shown that if $L$ is a compact distributive topological lattice of finite breadth and $U$ is a neighborhood of a point $x \in L$, then there is a closed interval $[y, z]$ that is a neighborhood of $x$ which lies in $U$. L. Anderson [1] has proved that if $L$ is a connected topological lattice and if $a \in L$, then

$$
a \wedge[L \mid(a \wedge L)] \subset F(a \wedge L)
$$

and dually. If $L$ is a locally compact, chain-wise connected distributive topological lattice, then $\operatorname{Br}(L) \leqq \mathrm{cd}(L)$ [3], where we say that $L$ is chain-wise connected if for each pair of elements $x$ and $y$ in $L$ with $x \leqq y$ there is a closed connected chain from $x$ to $y$. It is well known that a compact connected topological lattice is chain-wise connected.

Proof of 1.1. Suppose that $\mathrm{cd}(L)$ is finite. Then, by Anderson's result, we have that $\operatorname{Br}(L)$ is also finite. Since $L$ is compact, $L$ has a zero element 0 and a unit element 1 , and we can find a neighborhood $U$ of 0 such that $1 \notin U$. Now by Dyer and Shields' result, there exists a closed interval $[y, z]$ that is a neighborhood of 0 
which lies in $U$. However, $0 \in[y, z]$ implies $y=0$. Hence there exists an open subset $V$ such that $0 \in V \subset[0, z]=z \wedge L \subset U$. Since $L$ is nondegenerate and connected, we have $z>0$ and $z \neq 1$. Now by Anderson's result, we have that for the element $z>0$

$$
z \wedge[L \mid(z \wedge L)] \subset F(z \wedge L)
$$

By hypothesis, there exists a nonzero disjoint element $u$ of $z$. Clearly $u \in$ $L \mid(z \wedge L)$. Therefore $0 \in F(z \wedge L)$. On the other hand, $0 \in V \subset z \wedge L$ implies that $0 \notin F(z \wedge L)$. So we have a contradiction. Hence $c d(L)$ must be infinite.

Immediately, we have the following corollary:

1.2 Corollary. A compact and connected Boolean topological lattice $L$ is finite codimensional if and only if $L$ is degenerate.

It is easy to see that in a compact Boolean lattice, the continuities of $\wedge$ and $\vee$ imply the continuity of the unary operation of the complementation. So compact Boolean lattice forms always a semisimple compact topological ring. For these reasons, Kaplansky's theorem (Theorem 10 in [11]) implies that any compact Boolean lattice is iseomorphic to a cartesian directed sum of the Boolean lattices 2, where $2(=\{0,1\})$ has the discrete topology. Hence we have the following:

\subsection{TheOREM. Any compact Boolean topological lattice is totally disconnected.}

However, in the proof of Theorem 10 [11], Kaplansky used the character group property in the sense of topological groups (the so-called duality theorem). A. D. Wallace has suggested the possibility of a proof of (1.3) which does not involve the use of the duality theorem. In this connection, we prove the following:

1.4 Corollary. Any compact Boolean topological lattice of finite codimension is totally disconnected.

Proof. Let $L$ be a compact Boolean topological lattice of finite codimension and let $C$ be the component of 0 . It is easy to see that $C$ is a compact sublattice of $L$. So $C$ has a maximal element $m$. We show $C=m \wedge L$. That $C \subset m \wedge L$ is obvious. If $x \in m \wedge L$, then $x \wedge C$ is connected and $0 \in x \wedge C$. So $x=x \wedge m \in x \wedge C \subset C$ and hence $m \wedge L \subset C$. It is known that any Boolean lattice is relatively complemented. So a closed interval $C=m \wedge L$ is a compact connected Boolean topological lattice under its relative topology. It is also true that if $X$ is a topological space with $\operatorname{cd}(X)=n$ and if $C$ is either an open or a closed subset of $X$, then $\operatorname{cd}(C) \leqq n$ [15]. Therefore, $C$ is a compact connected Boolean topological lattice of finite codimension. By (1.2), our only possibility is that $C$ consists of the single element 0 . Since $L$ is a topological group, it is homogeneous, and hence $L$ is totally disconnected, which completes the proof.

If we are allowed to use the duality theorem, we can replace the compactness hypothesis in (1.3) by suitable local properties of $L$. 
We recall that if $L$ is a topological lattice and if for an arbitrary neighborhood $U$ of an element $x$ in $L$ there exists a convex open neighborhood $V$ of $x$ such that $V \subset U$, then $L$ is called locally convex [1], [12]. A subset $S$ of a topological $\operatorname{ring} A$ is bounded if for any neighborhood of the zero 0 of $A$ there exists a neighborhood of 0 such that $V S \subset U$, where $V S=\{v s \mid v \in V$ and $s \in S\}$. In [11], Kaplansky proved, using a character group property of a topological group, that if $C$ is the component of 0 in a bounded locally compact topological ring $A$, then $C A=\{0\}$.

1.5 LEMMA. If $L$ is a Boolean topological lattice and if $L$ is locally convex, then $L$ is bounded.

Proof. Let $U$ be a neighborhood of 0 . Then there exists a locally convex open neighborhood $V$ of 0 such that $V \subset U$. Clearly, by the convexity of $V, V \wedge L \subset V$. Hence $L$ is bounded because the operation $\wedge$ is the multiplicative operation in the Boolean ring.

1.6 THEOREM. If $L$ is a locally compact and locally convex topological Boolean lattice, then $L$ is totally disconnected.

Proof. By (1.5), $L$ is bounded. By Kaplansky's result, if $C$ is the component of 0 , then $C \wedge L=\{0\}$. If $x \in C$, then $x=x \wedge x \in C \wedge L=\{0\}$; hence $C=\{0\}$. But $L$ is still a topological group, and hence $L$ is totally disconnected.

We now exhibit a sufficient condition for a compact complemented modular topological lattice to be totally disconnected.

In a lattice, two closed intervals $[a \wedge b, a]$ and $[b, a \vee b]$ are called transposes of each other [6]. Two closed intervals, $[a, b]$ and $[c, d]$, are projective [6] if and only if there exists a finite number of closed intervals $\left[x_{i}, y_{i}\right]$ such that $\left[x_{i-1}, y_{i-1}\right]$ and $\left[x_{i}, y_{i}\right]$ are transposes, $i=1,2, \ldots, n$, where $[a, b]=\left[x_{0}, y_{0}\right]$ and $\left[x_{n}, y_{n}\right]=[c, d]$.

1.7 LemMa. If $L$ is a modular topological lattice, then any pair of projective intervals are topologically equivalent.

Proof. We need to prove only that two transposes $[a \wedge b, a]$ and $[b, a \vee b]$ are topologically equivalent. Define a mapping $f$ from $[a \wedge b, a]$ into $[b, a \vee b]$ by $f(x)=x \vee b$, and define a mapping $g$ from $[b, a \vee b]$ into $[a \wedge b, a]$ by $g(x)=a \wedge y$. Clearly, $f$ and $g$ are both continuous. Furthermore $g=f^{-1}$.

1.8 LEMMA. In a topological lattice with 0 , the following are equivalent, if $a \in L$ :

(i) $a \wedge L \neq F(a \wedge L)$,

(ii) $(a \wedge L)^{\circ} \neq \square$,

(iii) $0 \in(a \wedge L)^{\circ}$

and dually.

Proof. (i) $\rightarrow$ (ii) is obvious.

(ii) $\rightarrow$ (iii): Anderson has shown [1] that in a topological lattice $(a \wedge L) \vee F(a \wedge L)$ $=F(a \wedge L)$ for all $a \in L$. Suppose that there exists an element $x$ such that $x \in(a \wedge L)^{\circ}$ 
and $0 \notin(a \wedge L)^{\circ}$. It follows that $0 \in F(a \wedge L)$ because $0 \in a \wedge L$. However, $x=x \vee 0$ $\in(a \wedge L) \vee F(a \wedge L)=F(a \wedge L)$, which is a contradiction. Hence, $0 \in(a \wedge L)^{\circ}$.

(iii) $\rightarrow$ (i) is trivial.

For each element $x$ of a topological lattice $L$ with 0 and 1 we define $M(x)$ and $L(x)$ to be, respectively,

$$
M(x)=x \vee L(=[x, 1]) \text { and } L(x)=x \wedge L(=[0, x]) .
$$

If $L$ is a compact complemented modular topological lattice, then clearly each of the sublattices $M(x)$ and $L(x)$ is also a compact complemented modular topological lattice in its relative topology.

If $A$ and $B$ are subsets of $M(x)$ and $L(x)$, respectively, we use $F_{M}(A)$ and $F_{L}(B)$ to denote the relative boundaries of $A$ and $B$ respectively, and for $p \in M(x)(p \in$ $L(x))$ we note that $p \wedge M(x)=[x, p](p \vee L(x)=[p, x])$.

1.9 LEMMA. If $L$ is a compact complemented modular topological lattice and if for every nonzero element a of $L, a \wedge L \neq F(a \wedge L)$ and dually, then for every nonunit element $x$ and every element $p(\neq x)$ of $M(x), p \wedge M(x) \neq F_{M}(p \wedge M(X))$ and dually.

Proof. Since $x$ is not the unit element of $L$, a complement $y$ of $x$ is not the zero element. Hence, the two closed intervals $[0, y]$ and $[x, 1]$ are transposes. So, by Lemma (1.7), $[0, y]$ and $[x, 1]$ are topologically equivalent under the mapping $f:[0, y] \rightarrow[x, 1]$ defined by $f(a)=a \vee x$. Now if $p \in M(x) \mid x$ and $u \in[0, p \wedge y]$, then $f(u)=u \vee x \in[x, p]$. Therefore, $f([0, p \wedge y]) \subset[x, p]$. Conversely, if $z \in[x, p]$, then $f(y \wedge z)=x \vee(y \wedge z)=z$ by the modularity of $L$, and $y \wedge z \in[0, p \wedge y]$. Hence, $f([0, p \wedge y])=[x, p]$. Since $p \neq x$ and $p$ is the image of $p \wedge y$ under $f$, we have $p \wedge y \neq 0$. Now $((p \wedge y) \wedge L)^{\circ} \neq \square$ in $L$ by hypothesis and Lemma (1.8). Therefore, $((p \wedge y) \wedge L)^{\circ} \neq \square$ in $[0, y]$. Thus $[0, p \wedge y]=(p \wedge y) \wedge L$ implies $(f([0, p \wedge y]))^{\circ} \neq \square$ in $M(x)(=[x, 1])$. Applying Lemma $(1.8)$ to $M(x)$, we have that in $M(x)$, $p \wedge M(x) \neq F_{M}(p \wedge M(x))$ for each $p(\neq x) \in M(x)$. Dually, in $L(x), q \vee L(x)$ $\neq F_{M}(q \vee L(x))$ for each $q(\neq x) \in L(x)$. Thus the proof is complete.

1.10 LemMA. If $L$ is a compact complemented modular topological lattice and if for every nonzero element a of $L, a \wedge L \neq F(a \wedge L)$ and dually, then the components of the zero and the unit consist of the single elements $\{0\}$ and $\{1\}$, respectively.

Proof. Let $C$ be the component of 0 . We have already shown that $C$ is a compact topological lattice in its relative topology, and $C=p \wedge L$, where $p$ is the maximal element of $C$. Therefore, $C$ is also a connected complemented topological lattice in its relative topology since any sublattice of a complemented modular lattice is also relatively complemented. Suppose $p \neq 0$. Since there exists an arc from $p$ to 0 , we can find an element $a$ such that $0<a<p$. Applying Lemma 6 in [1] to $C$ we have that $a \in C$ implies $a \wedge[C \mid(a \wedge C)] \subset F_{C}(a \wedge C)$. Let $b$ be a relative complemented 
element of $a$ in $C=[0, p]$. Thus $b \neq 0$, and therefore $b \in C \backslash(a \wedge C)$, since if $b \in a \wedge C$, we have $b \leqq a$. Consequently,

$$
0=a \wedge b \in a \wedge[C \backslash(a \wedge C)] \subset F_{C}(a \wedge C) .
$$

On the other hand, by hypothesis, $0 \in[0, a]^{\circ}=(a \wedge C)^{\circ}$, which is a contradiction. Hence, $p$ must be the zero. It follows that $C$ consists of the single element 0 .

Dually, the component of 1 consists of the single element 1 .

Now we prove the following theorem:

1.11 THEOREM. If $L$ is a compact complemented modular topological lattice and if $a \wedge L \neq F(a \wedge L)$ for all nonzero elements $a$ of $L$ and dually, then $L$ is totally disconnected.

Proof. By applying Lemma (1.10) to $M(x)(x \neq 1)$ and $L(y)(y \neq 0)$, the components of $x$ and $y$ in $M(x)$ and $L(y)$ are the singletons $\{x\}$ and $\{y\}$, respectively.

Now consider the component $E_{x}$ of $x$ in $L$ where $x \neq 1$ and $x \neq 0$. We claim that $E_{x} \cap(x \vee L)=\{x\}$ and $E_{x} \cap(x \wedge L)=\{x\}$. In fact, if $y \in E_{x} \cap(x \vee L)$ and $y \neq x$, then $y>x$. Since $E_{x}$ is connected, $x \vee E_{x}$ is connected and contains both $x$ and $y$. On the other hand, $x \vee E_{x} \subset x \vee L \subset M(x)$, which is a contradiction. Hence $E_{x} \cap(x \vee L)=\{x\}$. Dually, $E_{x} \cap(x \wedge L)=\{x\}$. Consequently, $E_{x}$ consists of incomparable elements of $L$. For, if $a$ and $b$ are in $E_{x}$ and if $a \leqq b$, then $E_{x}=E_{a}=E_{b}$. Since $x$ and $a$ are incomparable, $a$ is neither the zero nor the unit element of $L$. Clearly, $b \in E_{b} \cap(a \vee L)=E_{a} \cap(a \vee L)=\{a\}$. Therefore, we have $a=b$. Now we will show that $E_{x}$ consists of the singleton $\{x\}$ in $L$. Suppose Card $\left(E_{x}\right)>1$. It follows that there exists an element $a$ of $E_{x}$ such that $a$ and $x$ are incomparable, and hence, $x \vee a \neq a$, i.e., $x \vee a>a$. Since $x \vee E_{x}$ is a connected set containing $x, x \vee E_{x} \subset E_{x}$. However, $x \vee a \in x \vee E_{x} \subset E_{x}$, which is contrary to the fact that $E_{x}$ consists of incomparable elements. Hence, $E_{x}=\{x\}$ for all nonzero nonunit elements $x$ of $L$. For the zero and the unit elements we have already shown in (1.10) that their components consist of the singletons $\{0\}$ and $\{1\}$, respectively. Hence, the proof is complete.

2. Compact and connected topological lattices. We recall that an element $a$ of a lattice $L$ is neutral if and only if every triple $\{a, x, y\}$ generates a distributive sublattice of $L$, and we also recall that an element $a$ is center element if and only if it is neutral and complemented in $L$. We shall write $\operatorname{Cen}(L)=\{x \mid x$ is a center element $\}$ and read "the center of $L$ ".

Now we prove Wallace's conjecture [14] which is stated in the introduction of this paper.

The following lemma is obvious.

2.1 LEMMA. If $L$ is a topological lattice and if $M$ is a distributive sublattice of $L$, then $M^{*}$ is also a distributive sublattice of $L$.

So any maximal distributive sublattice of a topological lattice is closed. And we recall that the set $N$ of all neutral elements is the intersection of all maximal 
distributive sublattices of the lattice (see [6]). Therefore $N$ is always a closed sublattice of the topological lattice.

The following lemma follows from the proof of Theorem 10 in [11].

2.2 Lemma. If $L$ is a totally disconnected compact Boolean topological lattice, then $L$ is iseomorphic with the set of all subsets of a set $X$ denoted by $P(X)$.

2.3 THEOREM. If $L$ is a compact and connected topological lattice and if $\mathrm{cd}(L)=n$, then $\operatorname{Card}(\operatorname{Cen}(L)) \leqq 2^{n}$.

Proof. Let $S$ be the set of all center elements in $L$. It is well known that $S$ forms a Boolean sublattice with 0 and 1 in the set $N$ of all neutral elements of $L$. Clearly, $N$ is a compact topological lattice in its relative topology. Now we show that $S$ is a closed subset of $N$. In fact, we may define mappings

$$
\phi: N \times N \rightarrow N \times N \text { by } \phi(x, y)=(x \wedge y, 0)
$$

and

$$
\psi: N \times N \rightarrow N \times N \text { by } \psi(x, y)=(1, x \vee y) .
$$

Obviously, $\phi$ and $\psi$ are both continuous. Therefore, it follows that $\phi^{-1}(\Delta) \cap \psi^{-1}(\Delta)$ is closed in $N \times N$, where $\Delta=\{(x, x) \mid x \in N\}$. Let $\pi$ be the projection mapping of $N \times N$ onto the first $N$. It is easy to see that $S=\pi\left(\phi^{-1}(\Delta) \cap \psi^{-1}(\Delta)\right)$. $N$ being compact, $S$ is closed. Hence $S$ is a compact Boolean topological lattice of finite codimension. By (1.4) $S$ is a totally disconnected compact Boolean topological lattice. It follows from Lemma (2.2) that $S$ is iseomorphic with $P(X)$ for some set $X$. Suppose that Card $(X)>n$. We can take, then, $(n+1)$ elements $a_{1}, a_{2}, \ldots, a_{n+1}$ in $S$ such that the iseomorphism from $S$ to $P(X)$ maps $a_{i}$ to an atom of $P(X)$. Thus, each $a_{i}$ is also an atom in $S$. Since $L$ is compact and connected, we can find a compact connected chain $C_{j}$ from 0 to $a_{i}$ in $L, i=1,2, \ldots, n+1$. It follows from $a_{i} \wedge a_{j}=0(i \neq j)$ that if $x_{i} \in C_{i}$ and $x_{j} \in C_{j}(i \neq j)$, then $x_{i} \wedge x_{j}=0$. Now consider the following mappings:

$$
\begin{array}{r}
f: C_{1} \times C_{2} \times \cdots \times C_{n+1} \rightarrow L \text { defined by } \\
f\left(x_{1}, \ldots, x_{n+1}\right)=x_{1} \vee \cdots \vee x_{n+1} \\
g: \begin{array}{r}
f\left(C_{1} \times C_{2} \times \cdots \times C_{n+1}\right) \rightarrow C_{1} \times C_{2} \times \cdots \times C_{n+1} \text { defined by } \\
g(x)=\left(x \wedge a_{1}, x \wedge a_{2}, \ldots, x \wedge a_{n+1}\right) .
\end{array}
\end{array}
$$

Clearly, both $f$ and $g$ are well defined and continuous. Furthermore $g=f^{-1}$ because $a_{i}(i=1,2, \ldots, n+1)$ are neutral elements. Thus $L$ contains a closed subset that is homeomorphic to a cartesian product $C_{1} \times \cdots \times C_{n+1}$ of nondegenerate compact connected chains $C_{i}$, which has codimension $(n+1)$ [7]. This is a contradiction. Hence, $\operatorname{Card}(X) \leqq n$. It follows that $\operatorname{Card}(\operatorname{Cen}(L)) \leqq 2^{n}$. This completes the proof.

We are now concerned with a representation of a distributive topological lattice by an $n$-cell. 
The set of $n$ elements $x_{1}, x_{2}, \ldots, x_{n}$ in a lattice $L$ is independent over an element $a$ of $L$ if and only if $x_{i}>a$ and $\left(x_{1} \vee \cdots \vee x_{k}\right) \wedge x_{k+1}=a, k=1,2, \ldots, n-1$ [6]. It is easy to see that if $L$ is distributive, then $x_{1}, \ldots, x_{n}$ are independent over $a$ if and only if $x_{i} \wedge x_{j}=a(i \neq j)$.

We will prove the following useful lemma:

2.4 LEMMA. If $L$ is a distributive topological lattice with 0 and 1 and if L contains $n$ independent elements $x_{1}, \ldots, x_{n}$ over 0 whose union is 1 , then $L$ is iseomorphic with the topological lattice of the cartesian product $\left(x_{1} \wedge L\right) \times \cdots \times\left(x_{n} \wedge L\right)$.

Proof. If we define a mapping $h: L \rightarrow\left(x_{1} \wedge L\right) \times \cdots \times\left(x_{n} \wedge L\right)$ by $h(a)$ $=\left(a \wedge x_{1}, \ldots, a \wedge x_{n}\right)$, then $h$ is obviously well defined and continuous. Furthermore, $h$ is a lattice-homomorphism.

Now we define a mapping $g:\left(x_{1} \wedge L\right) \times \cdots \times\left(x_{n} \wedge L\right) \rightarrow L$ by $g\left(a_{1}, \ldots, a_{n}\right)$ $=a_{1} \vee a_{2} \vee \cdots \vee a_{n}$. Then, clearly, $g$ is continuous. Furthermore, $g=h^{-1}$. Hence, $L$ is iseomorphic with $\left(x_{1} \wedge L\right) \times\left(x_{2} \wedge L\right) \times \cdots \times\left(x_{n} \wedge L\right)$.

2.5 Theorem. Suppose $L$ is a topological lattice with 0 and 1 . Then $L$ is iseomorphic with an $n$-cell if and only if $L$ satisfies the following two conditions:

(i) $L$ is distributive and contains $n$ independent elements $x_{1}, x_{2}, \ldots, x_{n}$ over 0 whose union is 1 .

(ii) Each $x_{i} \wedge L$ is a separable connected locally convex chain.

Proof. Suppose that there exists an iseomorphism $f$ from $L$ to an $n$-cell $I^{n}$ where $I$ is the real unit interval $[0,1]$. Then obviously, $L$ is distributive, since so is $I^{n}$. Consider the fundamental base of $I^{n}:(1,0,0, \ldots, 0),(0,1,0, \ldots, 0), \ldots$, $(0,0, \ldots, 1)$. These elements are clearly independent elements over the zero $(0, \ldots, 0)$ in $I^{n}$.

Let $x_{1}, \ldots, x_{n}$ be elements of $L$ for which $f\left(x_{1}\right)=(1,0, \ldots, 0), \ldots, f\left(x_{n}\right)$ $=(0,0, \ldots, 0,1)$. Since $f$ is a lattice isomorphism, these elements $x_{1}, \ldots, x_{n}$ are obviously independent elements over 0 in $L$, and moreover, for each $i$,

$$
f\left(x_{i} \wedge L\right)=\left\{\left(0, \ldots, 0, a_{i}, 0, \ldots, 0\right) \in I^{n} \mid a_{i} \in I\right\},
$$

and hence, $f\left(x_{i} \wedge L\right)$ is iseomorphic with $I$ for each $i$. It follows that each $x_{i} \wedge L$ is a separable connected locally convex chain.

Conversely, suppose that $L$ satisfies the conditions (i) and (ii) in the theorem. It follows from Lemma (2.4) that $L$ is iseomorphic with the cartesian product $\left(x_{1} \wedge L\right) \times \cdots \times\left(x_{n} \wedge L\right)$. By (ii) each $x_{i} \wedge L$ is a separable connected locally convex chain. Now we recall Anderson's result, [5], that any separable connected locally convex chain is order homeomorphic to the closed interval $I$. Consequently, it follows that $L$ is iseomorphic with $I \times \cdots \times I=I^{n}$, which completes the proof.

2.6 Corollary. If $L$ is a topological lattice with 0 and 1 with $\mathrm{cd}(L)=n$, then $L$ is iseomorphic with an $n$-cell if and only if L satisfies (i) in Theorem (2.5) and (ii) each $x_{i} \wedge L$ is separable, connected and locally compact. 
Before we prove the corollary we give the following lemma:

2.7 LemMA. If $L$ is a locally compact topological lattice with $\mathrm{cd}(L)=n$ and if $C_{1}, C_{2}, \ldots, C_{m}$ are nondegenerate locally compact connected chains, then

$$
\operatorname{cd}\left(L \times C_{1} \times \cdots \times C_{m}\right)=n+m .
$$

This lemma is an immediate consequence of $\mathrm{H}$. Cohen's results [7] that

(i) If $X$ is a locally compact Hausdorff space and if ind $(X)=1$, then $\operatorname{cd}(X)=1$, where "ind" means the inductive dimension of Urysohn and Menger [10], and

(ii) if $X$ and $Y$ are locally compact Hausdorff spaces and if $\operatorname{cd}(X)=n$ and ind $(Y)=1$, then $\mathrm{cd}(X \times Y)=n+1$.

Therefore, Lemma (2.7) follows immediately from (ii) since ind $\left(C_{i}\right)=1$ and the $C_{i}$, $i=1,2, \ldots, m$, are a locally compact chain.

Proof of Corollary 2.6. The necessity is obvious. We now prove the sufficiency. By Lemma (2.4), $L$ is iseomorphic with $\left(x_{1} \wedge L\right) \times \cdots \times\left(x_{n} \wedge L\right)$. Since $x_{i} \wedge L$ is nondegenerate, connected, and locally compact, we have $\mathrm{cd}\left(x_{i} \wedge L\right) \geqq 1$ for each $i$, and since $\operatorname{cd}(L)=\operatorname{cd}\left(\left(x_{1} \wedge L\right) \times \cdots \times\left(x_{n} \wedge L\right)\right)=n$, we can verify that each $\operatorname{cd}\left(x_{i} \wedge L\right)=1, i=1,2, \ldots, n$. Indeed, suppose $\mathrm{cd}\left(x_{i} \wedge L\right)>1$ for some $i$. For each $j(\neq i)$, we take a compact connected chain $C_{j}$ such that $C_{j} \subset x_{j} \wedge L$ and $\operatorname{cd}\left(C_{j}\right)=1$. Clearly, by Lemma (2.7), we have that

$$
\operatorname{cd}(L) \geqq \operatorname{cd}\left(C_{1} \times \cdots \times C_{l-1} \times\left(x_{i} \wedge L\right) \times C_{i+1} \times \cdots \times C_{n}\right)>n,
$$

which is a contradiction. Hence, cd $\left(x_{i} \wedge L\right)=1$ for each $i$, and hence, each $x_{i} \wedge L$ is a chain [1]. On the other hand, $x_{i} \wedge L$ is locally convex since it is connected and locally compact [4]. Therefore, by Theorem (2.5), $L$ is iseomorphic with an $n$-cell. Thus, the proof is complete.

We now consider the cardinality of the center in a distributive lattice with 0 and 1 . The following lemma is useful in the sequel.

2.8 Lemma. Let $L$ be a distributive topological lattice with 0 and 1 . If $x_{1}, \ldots, x_{n}$ $(n>1)$ are all atoms of the center in L and if $x$ and $y$ are two incomparable elements in $x_{i} \wedge$ L for some $i$, then

$$
\operatorname{Card}(\operatorname{Cen}(L))<\operatorname{Card}\left(\operatorname{Cen}\left(\left[x \wedge y, x \vee y \vee c\left(x_{\imath}\right)\right]\right)\right) \text {, }
$$

where $c\left(x_{i}\right)$ is the complemented element of $x_{i}$ in the center of $L$.

Proof. Without losing any generality, we can assume that $x$ and $y$ are two incomparable elements in $x_{1} \wedge L$. Clearly, Card $(\operatorname{Cen}(L))=2^{n}$. Now we shall show that the number of center elements in the closed interval $J=\left[x \wedge y, x \vee y \vee c\left(x_{1}\right)\right]$ is greater than $2^{n}$. To do so, consider the following pairs of center elements in $J$ : 
$\left(x, y \vee c\left(x_{1}\right)\right)$

$\left(x \vee x_{2}, y \vee x_{3} \vee x_{4} \vee \cdots \vee x_{n}\right),\left(x \vee x_{3}, y \vee x_{2} \vee x_{4} \vee \cdots \vee x_{n}\right)$, $\ldots,\left(x \vee x_{n}, y \vee x_{2} \vee \cdots \vee x_{n-1}\right)$

$\left(x \vee x_{2} \vee x_{3}, y \vee x_{4} \vee \cdots \vee x_{n}\right),\left(x \vee x_{2} \vee x_{4}, y \vee x_{3} \vee x_{5} \vee \cdots \vee x_{n}\right)$, $\ldots,\left(x \vee x_{n-1} \vee x_{n}, y \vee x_{1} \vee \cdots \vee x_{n-2}\right)$

$\left(x \vee x_{3} \vee \cdots \vee x_{n}, y \vee x_{2}\right),\left(x \vee x_{2} \vee x_{4} \vee \cdots \vee x_{n}, y \vee x_{3}\right)$, $\ldots,\left(x \vee x_{2} \vee \cdots \vee x_{n-1}, y \vee x_{n}\right)$

$\left(x \vee c\left(x_{1}\right), y\right)$.

Obviously, the number of all pairs is

$$
\left(\begin{array}{c}
n-1 \\
0
\end{array}\right)+\left(\begin{array}{c}
n-1 \\
1
\end{array}\right)+\cdots+\left(\begin{array}{l}
n-1 \\
n-1
\end{array}\right)=2^{n-1}
$$

Since $L$ is distributive and $x_{\imath} \wedge x_{j}=0(i \neq j)$ and $x_{i} \wedge y=x_{i} \wedge x=0(i \neq 1)$, it is not hard to see that not only are these pairs all distinct, but also all elements of these pairs are distinct from each other and any two elements in a pair are complements of each other in $J$. Moreover, $x$ and $y$ are different from both $x \wedge y$ and $x \vee y \vee c\left(x_{1}\right)$ since $x$ and $y$ are incomparable. Also, any element in a pair is different from both $x \wedge y$ and $x \vee y \vee c\left(x_{1}\right)$. Consequently, adjoining the pair $\left(x \wedge y, x \vee y \vee c\left(x_{1}\right)\right)$ of the trivial center elements in $J$, we have $2^{n}+2$ center elements in $J$. Hence, we have

$$
\text { Card }(\operatorname{Cen}(L))=2^{n}<\operatorname{Card}(\operatorname{Cen}(J)) \text {, }
$$

which is what we wanted to prove.

Applying the above lemma, we can easily obtain the following result of Anderson [3]:

2.9 Corollary. $H_{1}: L$ is a connected distributive topological lattice with 0 and 1 ,

$\mathrm{H}_{2}$ : each closed chain in $\mathrm{L}$ is locally convex and separable,

$H_{3}:$ if $a \leqq b \leqq c$, then Card (Cen $(b \vee(c \wedge L)) \leqq \operatorname{Card}(\operatorname{Cen}(a \vee L))$,

$H_{4}:$ Card $(\operatorname{Cen}(L))=2^{n}$,

$C: L$ is topologically isomorphic with $I^{n}$.

Proof. Since the center $C$ in $L$ forms a Boolean sublattice of $L$, it follows from $H_{4}$ that there exist exactly $n$ atoms $x_{1}, x_{2}, \ldots, x_{n}$ of $C$. These elements $x_{1}, \ldots, x_{n}$ are independent elements over 0 in $L$. Suppose that $x_{i} \wedge L$ is not a chain for some $i$. Then we can find two incomparable elements $x$ and $y$ in $x_{i} \wedge L$. By Lemma (2.8), observing that $0 \leqq x \wedge y \leqq x \vee y \vee c\left(x_{i}\right)$, we have a contradiction to $H_{3}$. Thus each $x_{i} \wedge L$ is a chain, so by $H_{2}, x_{i} \wedge L$ is locally convex and separable. Furthermore, $x_{i} \wedge L$ is connected since $L$ is. Hence, by Theorem (2.5), $L$ is iseomorphic with an $n$-cell $I^{n}$. Thus the proof is complete.

We shall now show that the number of all atoms of the center, the number of all independent elements over an element, the breadth and the codimension in a distributive topological lattice with 0 and 1 are related. 
2.10 LEMMA. If $L$ is a locally compact, chain-wise connected, distributive topological lattice with 0 and 1 , then $\mathrm{Ca}(L) \leqq \mathrm{Br}(L) \leqq \operatorname{In}(L) \leqq \mathrm{cd}(L)$ provided these numbers are finite, where

$\mathrm{Ca}(L):$ the number of all atoms in the center in $L$,

$\operatorname{Br}(L)$ : the breadth of $L$,

In $(L)$ : the maximal number of independent elements over an element of $L$, and cd $(L)$ : the codimension of $L$.

Proof. (i) $\mathrm{Ca}(L) \leqq \mathrm{Br}(L)$. Consider the center $C$ in $L$ and the set of all its dual atoms $D=\left\{d_{1}, d_{2}, \ldots, d_{k}\right\}$, where $k=\mathrm{Ca}(L)$. It is obvious that $\operatorname{Inf} F \neq 0$ for any proper subset $F$ of $D$. But $\operatorname{Inf} D=0$. Hence $\operatorname{Br}(L) \geqq k$.

(ii) $\mathrm{Br}(L) \leqq \operatorname{In}(L)$. Let $\mathrm{Br}(L)=m$. By definition of the breadth, there exists a finite subset $F$ of $L$ such that $\operatorname{Inf} F \neq \operatorname{Inf} B$ for any subset of $F$ with $\operatorname{Card}(B) \leqq m-1$. So $F$ contains a subset $A$ with $\operatorname{Card}(A)=m$ such that $\operatorname{Inf} A \neq \operatorname{Inf} B$ for any proper subset $B$ of $A$, for, otherwise, if $A$ is an arbitrary subset of $F$ with Card $(A)=m$, then Inf $A=\operatorname{Inf} B$ for some $B \subset A$ with $\operatorname{Card}(B) \leqq m-1$. Therefore, since $\operatorname{Inf}(A \cup x)$ $=\operatorname{Inf}(B \cup x)=\operatorname{Inf} B^{\prime}$ for any $x \in F \backslash A$ and some $B^{\prime} \subset B \cup x$ with Card $\left(B^{\prime}\right) \leqq m-1$, we have finally, by repeating this method, $\operatorname{Inf} F=\operatorname{Inf} C$ for some $C \subset F$ with Card $(C) \leqq m-1$, which is a contradiction.

Let $A=\left\{a_{1}, a_{2}, \ldots, a_{m}\right\}$ be such a subset of $F$, and let $x_{i}=\operatorname{Inf}\left(A \mid a_{i}\right), i=1,2$, $\ldots, m$. Also, let $a=\operatorname{Inf} A$. Then $x_{i} \neq a, i=1,2, \ldots, m$ and $x_{i} \neq x_{j}$ if $i \neq j$. It is easy to see that $\left(x_{1} \vee \cdots \vee x_{k}\right) \wedge x_{k+1}=a, k=1,2, \ldots, m-1$, i.e., $\left\{x_{1}, \ldots, x_{m}\right\}$ are independent elements over $a$. Hence $\operatorname{Br}(L) \leqq \operatorname{In}(L)$.

(iii) In $(L) \leqq c d(L)$. Suppose In $(L)=n$. Let $\left\{x_{1}, \ldots, x_{n}\right\}$ be a set of elements over an element $a$, and let $b=x_{1} \vee \cdots \vee x_{n}$. Clearly, $M=a \vee(b \wedge L)$ is a distributive topological lattice under its relative topology. By Lemma (2.4), $M$ is iseomorphic with $\left(x_{1} \wedge(a \vee M)\right) \times \cdots \times\left(x_{m} \wedge(a \vee M)\right)$. Since $x_{i} \wedge(a \vee M)$ is nondegenerate and chain-wise connected, we have $c d\left(x_{i} \wedge(a \vee M)\right) \geqq 1$ and so $x_{i} \wedge(a \vee M)$ contains a nondegenerate compact connected chain. By Lemma (2.7),

$$
\operatorname{cd}(M)=\operatorname{cd}\left(x_{1} \wedge(a \vee M) \times \cdots \times x_{n} \wedge(a \vee M)\right) \geqq n .
$$

Hence, In $(L)=n \leqq \mathrm{~cd}(L)$ since $M$ is a closed subset of $L$.

Now we prove the following theorem:

2.11 THEOREM. If $L$ is a locally compact chain-wise connected distributive topological lattice with 0 and 1 , the following are equivalent:

(i) $\mathrm{Ca}(L)=\mathrm{cd}(L)=n$,

(ii) $\mathrm{Ca}(L)=n$ and for each atom $x_{i}$ of the center in $L, x_{i} \wedge L$ is a chain,

(iii) $L$ is iseomorphic with a cartesian product of $n$ nondegenerate compact connected chains.

Proof. (i) $\rightarrow$ (ii): Suppose $\mathrm{Ca}(L)=\mathrm{cd}(L)=n$. Let $\left\{x_{1}, \ldots, x_{n}\right\}$ be the set of all atoms of the center in $L$. By Lemma (2.4), $L$ is iseomorphic with $\left(x_{1} \wedge L\right) \times \cdots$ $\times\left(x_{n} \wedge L\right)$. So each $x_{i} \wedge L$ contains a nondegenerate connected closed chain. Hence, 
cd $\left(x_{i} \wedge L\right)=1$, and since $x_{i} \wedge L$ is locally compact and connected, each $x_{i} \wedge L$ is a closed connected chain.

(ii) $\rightarrow$ (iii): Suppose that $x_{i} \wedge L, i=1,2, \ldots, n$, are chains. Since $x_{i} \wedge L$ is locally compact and connected, it follows that $x_{i} \wedge L$ is locally convex [1]; therefore $x_{i} \wedge L$ is a nondegenerate compact connected chain [4]. Again by Lemma (2.4), $L$ is iseomorphic with a cartesian product of $n$ nondegenerate compact connected chains.

(iii) $\rightarrow$ (i): Suppose that $L$ is iseomorphic with $C_{1} \times \cdots \times C_{n}$ where $C_{i}$, $i=1,2, \ldots, n$, are compact connected chains with zero element $0_{i}$ and unit element $1_{i}$. Clearly, $\operatorname{cd}(L)=\mathrm{cd}\left(C_{1} \times \cdots \times C_{n}\right)=n$ [7]. It is easy to see that $\left(0_{1}, \ldots, 0_{i-1}, 1\right.$, $\left.0_{i+1}, \ldots, 0_{n}\right), i=1,2, \ldots, n$, are atoms of the center of $C_{1} \times \cdots \times C_{n}$. Therefore, $\mathrm{Ca}(L) \geqq n=\mathrm{cd}(L)$. Since $\mathrm{Ca}(L) \leqq \mathrm{cd}(L)$, we have $\mathrm{Ca}(L)=\mathrm{cd}(L)=n$. Thus, the proof is complete.

2.12 LEMMA. If $L$ is a modular topological lattice and if $X=[u \wedge v, u]$ and $Y=[u \wedge v, v]$ are two closed intervals in $L$, then the topological sublattice generated by $X$ and $Y$ is iseomorphic to the cartesian product of $X$ and $Y$.

Proof. Let $S$ be the topological sublattice generated by $X$ and $Y$. By Theorem 7 , p. 73, in [6], $S$ and $X \times Y$ are lattice isomorphic under the mapping $f: X \times Y \rightarrow S$ defined by $f(x, y)=x \vee y$. Clearly, $f$ is continuous. If we define a mapping $g: S \rightarrow X \times Y$ by $g(a)=(u \wedge a, v \wedge a)$, then $g$ is also continuous, and moreover, $g=f^{-1}$. Hence, $S$ and $X \times Y$ are iseomorphic.

One can easily extend Lemma (2.12) by induction as follows:

2.13 Corollary. If $x_{1}, \ldots, x_{n}$ are independent elements over $a$ in a modular topological lattice $L$, then the topological sublattice generated by the intervals $\left[a, x_{k}\right], k=1,2, \ldots, n$, is iseomorphic with the cartesian product of the $\left[a, x_{k}\right]$ 's, $k=1,2, \ldots, n$.

(See the corollary, p. 73 in [6].)

As an immediate consequence of Corollary (2.13), we have the following:

2.14 THEOREM. If $L$ is a locally compact chain-wise connected modular topological lattice, then $\operatorname{In}(L) \leqq \mathrm{cd}(L)$.

From the definition of the ordinal sum of two partially ordered sets [6], we are motivated to define the ordinal sum of two topological lattices $L_{1}$ with the unit $1_{1}$ and $L_{2}$ with the zero $0_{2}$ as follows:

The ordinal sum $L_{1} \oplus L_{2}$ is the subset $\left(L_{1} \times\left\{0_{2}\right\}\right) \cup\left(\left\{1_{1}\right\} \times L_{2}\right)$ of the cartesian product $L_{1} \times L_{2}$.

Clearly, $L_{1} \oplus L_{2}$ is also a topological lattice under its relative topology in $L_{1} \times L_{2}$.

Recall that an element $a$ of a connected topological space $L$ is a cut point if and only if $L \backslash a$ is not connected [18].

The following theorem appears in [4]. 
2.15 THEOREM. If $L$ is a connected topological lattice and if $p \in L$, then $p$ is a cut point of $L$ if and only if $p \neq 0, p \neq 1$, and $L=(p \wedge L) \cup(p \vee L)$.

2.16 Lemma. Let $L$ be a connected topological lattice and let $a \in L, a \neq 0$, and $a \neq 1$. $a$ is a cut point of $L$ if and only if there exists an iseomorphism $f: L \rightarrow(a \wedge L)$ $\oplus(a \vee L)$ such that $f(a)=(a, a)$.

Proof. Suppose that $a$ is a cut point of $L$. By Theorem (2.15), $L=(a \wedge L)$ $\cup(a \vee L)$. Now we may define a mapping $f: L \rightarrow(a \wedge L) \oplus(a \vee L)$ as the following:

$$
\begin{array}{ll}
f(x)=(a, x) & \text { if } x \in a \wedge L, \\
f(y)=(y, a) & \text { if } y \in a \vee L .
\end{array}
$$

Then clearly, $f$ is well defined, is a lattice-isomorphism, and is continuous and also $f(a)=(a, a)$. Again we define a mapping $g:(a \wedge L) \oplus(a \vee L) \rightarrow L$ as follows :

$$
\begin{array}{ll}
g(a, x)=x \vee a(=x) & \text { if }(a, x) \in\{a\} \times(a \vee L), \\
g(y, a)=a \wedge y(=y) & \text { if }(y, a) \in(a \wedge L) \times\{a\} .
\end{array}
$$

It is also easy to see that $g$ is well defined and continuous. Furthermore, $g=f^{-1}$.

Conversely, suppose that $L$ is iseomorphic with $(a \wedge L) \oplus(a \vee L)$ by a mapping $f$ with $f(a)=(a, a)$. By definition

$$
(a \wedge L) \oplus(a \vee L)=((a \wedge L) \times\{a\}) \cup(\{a\} \times(a \vee L)) \subset(a \wedge L) \times(a \vee L) .
$$

Clearly, the element $(a, a)$ is a cut point of $(a \wedge L) \oplus(a \vee L)$, so the element $a$ is a cut point of $L$. Thus, the proof is complete.

2.17 Lemma. Let $L$ be a compact connected distributive topological lattice with $\operatorname{cd}(L)=n$. If $\operatorname{Card}(\operatorname{Cen}([a, b])) \geqq \operatorname{Card}(\operatorname{Cen}([c, d]))$ for any $[c, d] \subset[a, b]$ in $L$, then the closed interval $[a, b]$ is iseomorphic with a cartesian product of $m$ compact connected chains $(m \leqq n)$.

Proof. Since $[a, b]=a \vee(b \wedge L),[a, b]$ is also a compact connected distributive topological lattice under its relative topology in $L$. Let us first suppose that Card $(\operatorname{Cen}([a, b]))=2$. It follows that $[a, b]$ is a chain because otherwise, there would exist two incomparable elements $x$ and $y$ in $[a, b]$ so that

$$
\text { Card }(\operatorname{Cen}([x \wedge y, x \vee y]))
$$

would be $>2$. Now suppose Card (Cen $([a, b]))=2^{n}$ where $m>1$. By Theorem (2.3) we see that $m \leqq n$. Let $x_{1}, x_{2}, \ldots, x_{m}$ be all the atoms of the center of $[a, b]$. By Lemma (2.4), $[a, b]$ is iseomorphic with $\left(x_{1} \wedge[a, b]\right) \times\left(x_{2} \wedge[a, b]\right) \times \cdots \times\left(x_{m} \wedge[a, b]\right)$. From Lemma (2.8) we can easily see that each $x_{i} \wedge[a, b]$ is a chain. On the other hand, each $x_{i} \wedge[a, b]$ is compact and connected. Hence, the proof is complete.

Now we prove the following theorem:

2.18 THEOREM. Let $L$ be a compact connected distributive topological lattice with $\operatorname{cd}(L)=n . \quad L$ is iseomorphic with $\left(C_{1} \times C_{2} \times \cdots \times C_{p}\right) \oplus\left(D_{1} \times D_{2} \times \cdots \times D_{m}\right)$ 
where $C_{i}$ and $D_{j}$ are closed connected chains, $i=1,2, \ldots, p, j=1,2, \ldots, m$, if and only if there exists a cut point a such that Card $(\operatorname{Cen}(a \wedge L)) \geqq \operatorname{Card}(\operatorname{Cen}([b, c]))$ whenever $b \leqq c \leqq a$, and Card $(\operatorname{Cen}(a \vee L)) \geqq \operatorname{Card}(\operatorname{Cen}([d, e]))$ whenever $a \leqq d \leqq e$. Moreover, $\operatorname{cd}(L)=\operatorname{Br}(L)=\max \{p, m\}$.

Proof. Suppose that $L$ is iseomorphic with $\left(C_{1} \times \cdots \times C_{p}\right) \oplus\left(D_{1} \times \cdots \times D_{m}\right)$ by a mapping $f$. By Lemma (2.13), $\left(\left(1_{1}, \ldots, 1_{p}\right),\left(0_{1}, \ldots, 0_{m}\right)\right)$ is a cut point of $\left(C_{1} \times \cdots \times C_{p}\right) \oplus\left(D_{1} \times \cdots \times D_{m}\right)$ where $1_{i}$ and $0_{j}$ are unit element of $C_{i}$ and zero element of $D_{j}$, respectively, $i=1,2, \ldots, p, j=1,2, \ldots, m$. So there exists a cut point $a$ in $L$ such that $f(a)=\left(\left(1_{1}, \ldots, 1_{p}\right),\left(0_{1}, \ldots, 0_{m}\right)\right)$ and $a \wedge L$ and $a \vee L$ are iseomorphic with $C_{1} \times \cdots \times C_{p}$ and $D_{1} \times \cdots \times D_{m}$, respectively. Clearly, it follows that Card $(\operatorname{Cen}(a \wedge L)) \geqq \operatorname{Card}(\operatorname{Cen}([b, c]))$ whenever $b \leqq c \leqq a$ and

$$
\text { Card }(\operatorname{Cen}(a \vee L)) \geqq \operatorname{Card}(\operatorname{Cen}([d, e]))
$$

whenever $a \leqq d \leqq e$.

Conversely, by Lemma (2.16), $L$ is iseomorphic with $(a \wedge L) \oplus(a \vee L)$ and by Lemma (2.17), $a \wedge L$ and $a \vee L$ are iseomorphic with a cartesian product $C_{1} \times C_{2}$ $\times \cdots \times C_{p}$ and $D_{1} \times D_{2} \times \cdots \times D_{m}$ of compact connected chains where $p \leqq n$ and $m \leqq n$. Hence, $L$ is iseomorphic with $\left(C_{1} \times C_{2} \times \cdots \times C_{p}\right) \oplus\left(D_{1} \times D_{2} \times \cdots \times D_{m}\right)$. On the other hand, $\operatorname{cd}\left(C_{1} \times \cdots \times C_{p}\right)=p$ and $\operatorname{cd}\left(D_{1} \times \cdots \times D_{m}\right)=m$. Therefore, $\operatorname{cd}(L)=\operatorname{Br}(L)=\max \{p, m\}$.

One can easily extend the definition of the ordinal sum of two topological lattices to arbitrary finite numbers of topological lattices as follows:

If $L_{i}$ is a topological lattice with the zero element $0_{i}$ and the unit element $1_{i}$, $i=1,2, \ldots, m$, then the ordinal sum of $L_{1}, L_{2}, \ldots, L_{m}$ is defined as a subset

$$
\bigcup_{i=1}^{m^{\prime}}\left(\left\{1_{1}\right\} \times\left\{1_{2}\right\} \times \cdots \times\left\{1_{i-1}\right\} \times L_{i} \times\left\{0_{i+1}\right\} \times \cdots \times\left\{0_{m}\right\}\right)
$$

of the cartesian product $L_{1} \times L_{2} \times \cdots \times L_{m}$.

Obviously, the ordinal sum operation $\oplus$ is associative up to iseomorphism but is not commutative.

One can also extend Theorem (2.18) as follows:

$2.18^{\prime}$ THEOREM. Let $L$ be a compact connected distributive topological lattice with $\operatorname{cd}(L)=n . L$ is iseomorphic with

$$
\left(A_{1} \times A_{2} \times \cdots \times A_{p}\right) \oplus\left(B_{1} \times B_{2} \times \cdots \times B_{q}\right) \oplus \cdots \oplus\left(D_{1} \times D_{2} \times \cdots \times D_{r}\right)
$$

where $A_{i}, B_{j}, \ldots, D_{k}$ are closed connected chains, $i=1,2, \ldots, p, j=1,2, \ldots, q, \ldots$, $k=1,2, \ldots, r$, if and only if there exists a finite chain $0=a_{0}<a_{1}<\cdots<a_{m}=1$ of cut points $a_{1}, a_{2}, \ldots, a_{m-1}$ of $L$ such that $\operatorname{Card}\left(\operatorname{Cen}\left(\left[a_{i-1}, a_{i}\right]\right)\right) \geqq \operatorname{Card}(\operatorname{Cen}([c, d]))$ whenever $a_{i-1} \leqq c \leqq d \leqq a_{i}, i=1,2, \ldots, m$. Moreover,

$$
\operatorname{cd}(L)=\operatorname{Br}(L)=\max \{p, q, \ldots, r\} \text {. }
$$

The following theorem which is due to Cohen appears in [7]: 
2.19 THEOREM (SUM THEOREM). If $X$ is a locally compact Hausdorff space and if $X$ is the union of a countable family of closed sets each in $D^{n}(G)$ (see \$2), then $X \in D^{n}(G)$.

Now we shall prove the following theorem:

2.20 THEOREM. If $L$ is a locally compact connected topological lattice and if $L$ is the union of a countable family of compact connected chains of $L$, then $L$ is a chain.

Proof. If $C$ is a nonvoid, nondegenerate compact connected chain of $L$, then $\mathrm{cd}(C)=1$. For, $C$ is a compact connected topological lattice under its relative topology, and hence $C$ has the order topology (see [4]). It follows that ind $(C)=1$. From a result of Cohen [7], we have $\mathrm{cd}(C)=1$. Therefore, $C \in D^{1}(G)$. By the Sum theorem, we obtain $L \in D^{1}(G)$. It follows that $\mathrm{cd}(L) \leqq 1$. By a result of Anderson [1], $L$ is a chain.

\section{BIBLIOGRAPHY}

1. L. W. Anderson, One dimension topological lattices, Proc. Amer. Math. Soc. 10 (1959), 715-720.

2. - On the breadth and codimension of topological lattice, Pacific J. Math. 9 (1959), $327-333$.

3. - Topological lattices and n-cells, Duke Math. J. 25 (1958), 205-208.

4. - On the distributivity and simple connectivity of plane topological lattices, Trans. Amer. Math. Soc. 91 (1959), 102-112.

5. —_ Topological lattices, Tulane Dissertation, New Orleans, La., 1956.

6. G. Birkhoff, Lattice theory, Amer. Math. Soc. Colloq. Publ., Vol. 25, Amer. Math. Soc., Providence, R. I., 1948; revised 1967.

7. H. Cohen, A cohomological definition of dimension for locally compact Hausdorff spaces, Duke Math. J. 21 (1954), 209-224.

8. E. Dyer and A. Shields, Connectivity of topological lattices, Pacific J. Math. 9 (1959), 443-448.

9. S. Eilenberg and N. Steenrod, Foundations of algebraic topology, Princeton Univ. Press, Princeton, N. J., 1952.

10. W. Hurewicz and H. Wallman, Dimension theory, Princeton Univ. Press, Princeton, N. J., 1948.

11. I. Kaplansky, Topological rings, Amer. J. Math. 69 (1947), 153-183.

12. L. Nachbin, Topology and order, Univ. of Chicago, Chicago, Ill., 1950.

13. A. D. Wallace, Factoring a lattice, Proc. Amer. Math. Soc. 9 (1958), 250-252.

14. - - A note on mobs, An. Acad. Brasil. Ci. 24 (1952), 329-334.

15. —_, An outline for algebraic topology. I, Tulane Univ., New Orleans, La., 1949.

16. L. E. Ward, Jr., Binary relation in topological space, An. Acad. Brasil. Ci. 26 (1954), 357-373.

17. - - Partially ordered topological spaces, Proc. Amer. Math. Soc. 5 (1954), 344-361.

18. G. T. Whyburn, Analytic topology, Amer. Math. Soc. Colloq. Publ., Vol. 28, Amer. Math. Soc., Providence, R. I., 1942; revised 1967.

UNIVERSITY OF FLORIDA,

GainesVille, Florida

UNIVERSITY OF MASSACHUSETTS,

Amherst, Massachusetts 